\title{
Changes in the distribution of F-latency (DFL) for different postures of head and neck
}

\author{
Sabrina Sharmin ${ }^{1}$ and K Siddique-e Rabbani \\ Department of Biomedical Physics \& Technology, University of Dhaka, Dhaka, Bangladesh \\ ${ }^{1}$ Presently at: Department of Arts \& Sciences, \\ Ahsanullah University of Science \& Technology, Dhaka, Bangladesh \\ Email: ssr_8219@yahoo.com, rabbani@du.ac.bd
}

\begin{abstract}
Distribution of F-Latency (DFL) is a new neurological parameter conceived by the Biomedical Physics group of Dhaka University. Previous experimental studies have indicated that DFL, obtained from median nerve, varies with postures of the subject, particularly with the bending of the head. It agreed with the contention that bending of head might produce compression or stretching of nerve fibers as they come out of the vertebra, and that DFL is a sensitive parameter that gets affected by even such temporary changes. The present work was taken up with two objectives, i) to improve upon the earlier work by taking measurements with a few specific angles of head bending, particularly in the left and right directions, and ii) to provide a verification of earlier results. For bending of head to left and right sides, we chose $30^{\circ}$ and $45^{\circ}$ respectively. For forward and backward directions the angles were not measured and the subjects were asked to bend as much as they could without going beyond a comfort level. The study was carried out on 6 student volunteers aged between 24 and 26. The DFLs obtained with different postures of the head were analyzed to look for systematic trends in the changes, if any. Changes in several parameters like peak, mean, standard deviation, skewness and half-width in the rising and falling edges (down to $50 \%$ height of the peak) were studied for different postures of head and neck which showed consistent changes.
\end{abstract}

Keywords: Nerve conduction, Distribution of F-Latency, DFL, Distribution of Conduction Velocity, DCV, Cervical Spondylosis.

\section{INTRODUCTION}

In the Biomedical physics Laboratory of Dhaka University a new nerve conduction parameter named Distribution of F-latencies (DFL) was introduced [Rabbani et al 2007], which was shown to give Distribution of Conduction Velocities (DCV) of motor nerve fibers in a peripheral nerve trunk through a mirror image. DFL is obtained as a frequency distribution of F-latencies through multiple supramaximal stimulations of a peripheral nerve and the relationship with DCV was based on an explanation based on physiology, physics and statistics.

DFL has been found to be a sensitive parameter in the detection of radiculopathy (compression of nerve roots near the vertebra) or myelopathy (compression of the spinal cord) in the cervical regions, even at a subclinical stage [Alam and Rabbani 2010, Hossain et al 2011, Rabbani et al 2014, Chowdhury et al 2014]. Routine clinical investigations carried out by the group also indicated similar patterns for radiculopathy in lumbosacral regions. DFL has a single peaked distribution for a normal healthy motor 
nerve while broad peaks, double or triple peaks were observed in patients with the above mentioned disorders. The reasons for these patterns have been explained based on the physiology and anatomy of radiculopathy and myelopathy [Rabbani 2011] which involve compression or partial degeneration of a segment of a peripheral nerve. DFL with a single peak has also been seen to shift to longer delays due to compression at the wrist (Carpal Tunnel Syndrome, CTS) [Rabbani et al 2007]. Therefore, any such physical change in the nerve pathway between the periphery and the spinal cord may affect DFL. Prolonged compression of a nerve trunk may have complex effects including complete destruction of certain nerve fibres which effectively reduces conduction velocity across the compressed site.

For the above research, DFL measurements were carried out extensively in the upper limbs of many subjects over the years. Although DFL obtained from a particular nerve of a single subject reproduces well with repeated measurements, sometimes variations are observed and one of the factors considered for such variation was the posture of the head which may have affected nerve conduction through the brachial plexus, through physical compression or extension. An exploratory study showed that changes indeed happen due to such postural changes [Rahman, 2007] and therefore a more systematic study was taken up in the present work so that the effect can be understood with greater insight.

\section{METHODS}

A Homemade computerized EMG equipment was designed and fabricated locally with initial expertise gained from the University of Sheffield, UK [Rabbani et al, 1989], this has been providing routine clinical service in Bangladesh through a non-government and non-profit R \& D organization since 1988, and was used for the measurements in these studies as well. The data acquisition software was modified appropriately for this study.

A supramaximal electric stimulus applied at practically any point along the course of a nerve elicits the Fwave. Placing the anode distal to the cathode or off the nerve trunk avoids anodal block of the antidromic impulse. A surface electrode placed over the motor point of the tested muscle serves as the active lead against the reference electrode over the tendon. An optimal display of F-waves requires an amplifier gain of 200 or $500 \mu \mathrm{V} / \mathrm{cm}$ and an oscilloscope sweep of 5 or $10 \mathrm{~ms} / \mathrm{cm}$, depending on the nerve length and stimulus point. The earlier time from stimulation to the onset of an F-response was chosen as the Flatency in the present work. The existing nerve stimulator used, had different intervals between stimulation pulses, we chose time interval of about $1 \mathrm{sec}$.

To do this research at first we selected six subjects from fellow students at Dhaka University with age between 24 and 26 years irrespective of any neurological history. With informed oral consent, they volunteered to participate in this study. The subjects came in for tests according to their convenience and were assigned subjects numbers serially. Instead of full name, codes of their names were used. Age, gender and nerve length were recorded. The experiments were performed on different days and at different time. However, they did not complain of any neurological disorder. The measurements were carried out at room temperature, which remained steady at or around $28{ }^{\circ} \mathrm{C}$.

The idea was to measure DFL on a few subjects for different bent positions of the head with the subject sitting on a chair. DFLs were measured on left median nerve for the following head positions: upright, tilted left at $30^{\circ}$ and $45^{\circ}$, tilted right at $30^{\circ}$ and $45^{\circ}$, bent backward and bent forward. For the side tilting 
measurements a piece of plywood with angular markings were fixed at the back of a chair. For bending towards back and front the angle was not specified; the subjects were asked to bend their heads as much as possible without straining themselves with the back piece taken out.

Six subjects (students) with age between 24 and 26 years were studied. For each DFL the median nerve was stimulated at the wrist electrically for about 30 times with intervals of about a second between stimulations while the corresponding evoked EMG responses was recorded from the Thenar muscle at the base of the thumb. F-waves were the secondary (delayed) responses which were present in most of the responses; some responses did not have any F-wave. The F-latencies were obtained as the interval between the stimulation and the onset of the F-waves. For each DFL the frequency of occurrence of Flatencies were obtained in successive $2 \mathrm{~ms}$ groups and a frequency polygon was plotted. To compare the changes in DFL patterns with different head positions, analyses were performed to observe the changes of DFL patterns with respect to that for the upright position of the head, taken as the reference by determining the latency at peak (Lat ${ }_{\text {peak }}$ ) and some statistical parameters.

The following steps were taken for carrying out this present work. The frequency distribution of F-latency were grouped in following ways:

i) Head tilted on the same side of stimulating hand (i.e. on the left side) at $30^{\circ}$ and $45^{\circ}$

ii) Head tilted on the opposite side of stimulating hand (i.e. on the right side) at $30^{\circ}$ and $45^{\circ}$

iii) Head tilted on backward and forward directions.

At each group, the DFLs obtained were compared with that of the normal position of head. After obtaining DFLs for each of the above mentioned groups, the following analyses were performed:

\section{1) Quantitative analysis:}

DFLs were analyzed quantitatively by doing some statistical calculation. Three statistical parameters, mean, standard deviation and skewness were calculated for DFL of each position.

\section{2) Qualitative analysis:}

Skewness identifies how the distribution deviates from symmetry, however, it does not always give a simple-to-interpret value as it depends on both the tail and the fatness of the curve.

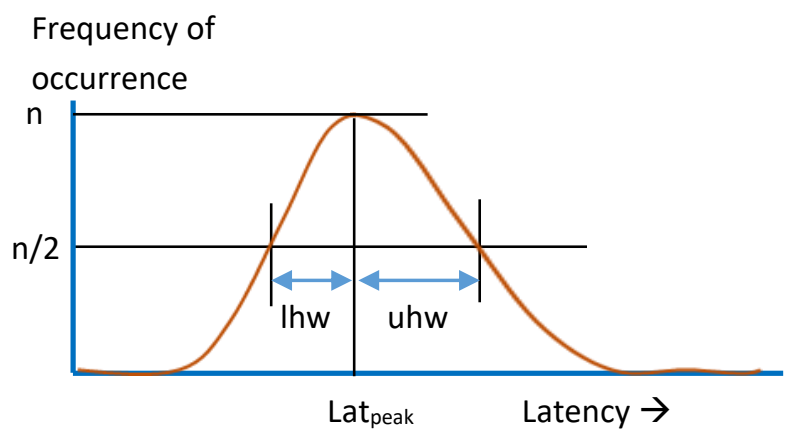

Figure.1: Defining lower and the upper half width 
Therefore, DFLs were analyzed qualitatively by finding the change in width at $50 \%$ peak height of DFL in the rising and falling edge and direction of the shifting of peak of DFL. The lower and the upper half width at half maximum as defined in Figure 1 (indicated by 'lhw' and 'uhw' respectively) essentially gives the asymmetry of the curve.

\section{3) Study on reproducibility of DFL:}

Since DFL is a statistically reproducible parameter for a nerve trunk of a subject, it is assumed to remain the same for repeated measurements for the same posture and needs to be experimentally verified. So, the reproducibility of DFL was also tested by repeating the experiment for a single subject at two different sessions.

The results are given in the following section.

\section{RESULTS AND OBSERVATIONS}

Typical DFL patterns obtained from one of the subjects (subject 6) are shown in Figure 2. The peak value occurs at a latency of $25 \mathrm{~ms}$ for the upright position while it is shifted to $27 \mathrm{~ms}$ for all other positions of head bending. This indicates that bending the head from normal position causes a delay in nerve conduction at the Brachial Plexus. It can further be seen that most F-latencies were concentrated within two $2 \mathrm{~ms}$-groups with mean values at $25 \mathrm{~ms}$ and $27 \mathrm{~ms}$, indicating that the F-latencies have a maximum range of about $4 \mathrm{~ms}$ (corresponding to maximum - minimum of these two groups respectively, i.e., $28 \mathrm{~ms}$ $-24 \mathrm{~ms})$. However, for the forward and backward bending, non-zero mean values of F-latency occurred at three consecutive values, at $25 \mathrm{~ms}, 27 \mathrm{~ms}$ and $29 \mathrm{~ms}$, which indicates that the minimum to maximum range increased to about $6 \mathrm{~ms}$. This indicates that the delay increased further in some of the nerve fibres on forward and backward bending.

The Lat ${ }_{\text {peak, }}$ Mean and Skewness calculated from the above DFLs (subject 6) are given in Table 1. Table 2 shows the direction of shifts of Lat ${ }_{\text {peak }}$ in all the 6 subjects. For Subject 6 the measurements were performed twice, to see if the measurements are consistent, which, is observed to be true for this parameter. 

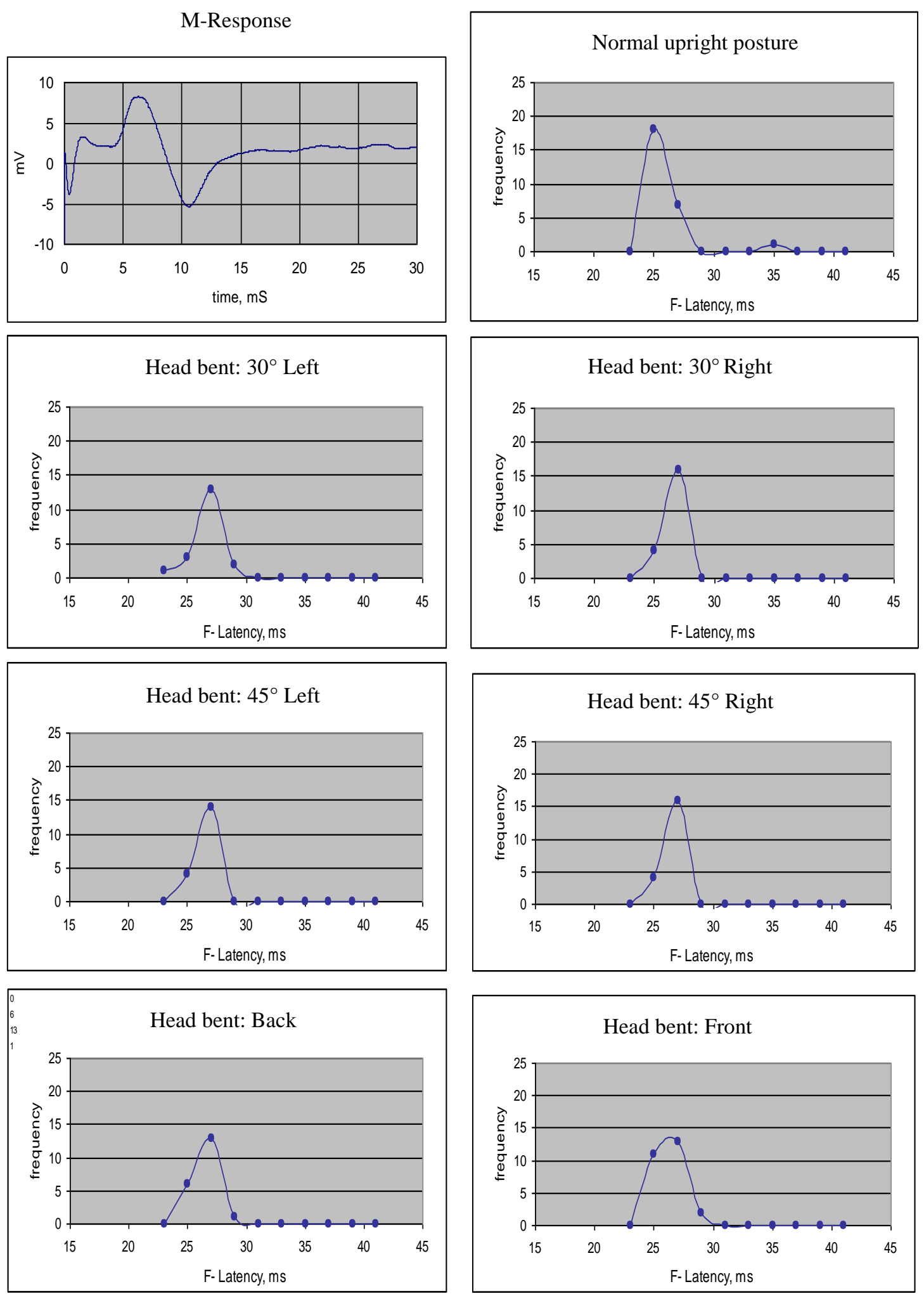

Figure 2: DFL obtained from the left median nerve of a subject (subject no. 6) for different postures of head. The graph in the top left corner shows the M-response 
Table-1: Three relevant parameters for subject 6 for two repeat trials taken on two different days. The deviation from those for the upright position is to be noted. Also it shows good reproducibility between the trials.

\begin{tabular}{|c|c|c|c|c|c|c|}
\hline \multirow{2}{*}{ Posture } & \multicolumn{2}{|c|}{ Latency peak, $m$ } & \multicolumn{2}{c|}{ Mean, $m s$} & \multicolumn{2}{c|}{ Skewness } \\
\cline { 2 - 7 } & Trial-1 & Trial-2 & Trial-1 & Trial-2 & Trial-1 & Trial-2 \\
\hline Upright & 25 & 25 & 26.12 & 25.82 & 4.11 & 1.57 \\
\hline $30^{\circ}$ Left & 27 & 27 & 26.47 & 26.02 & -1.97 & 1.39 \\
\hline $45^{\circ}$ Left & 27 & 27 & 26.75 & 26.14 & -0.26 & -0.33 \\
\hline $30^{\circ}$ Right & 27 & 27 & 26.18 & 26.44 & -0.69 & 0.47 \\
\hline $45^{\circ}$ Right & 27 & 27 & 26.42 & 26.15 & 0.94 & 0.41 \\
\hline Back & 27 & 27 & 26.30 & 26.08 & 0.78 & -1.49 \\
\hline Front & 27 & 27 & 26.20 & 26.39 & 1.16 & 0.37 \\
\hline
\end{tabular}

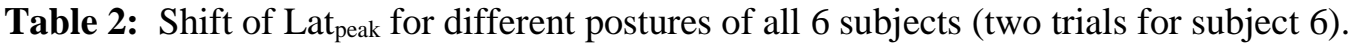
Peak shifted towards right, higher latency: $(\rightarrow)$, No change: $(N C)$, Peak shifted towards left, lower latency: $(\leftarrow)$. Respective reference values of Lat ${ }_{\text {peak }}$ are in $\mathrm{ms}$, for upright position of head.

\begin{tabular}{|c|c|c|c|c|c|c|c|}
\hline$\overbrace{\text { Posture }}^{\text {Subject }}$ & 1 & 2 & 3 & 4 & 5 & $6-a$ & $6-b$ \\
\hline $\begin{array}{c}\text { Upright } \\
\text { (reference) }\end{array}$ & 31 & 31 & 33 & 27 & 33 & 25 & 25 \\
\hline $30^{\circ} \mathrm{Left}$ & $\rightarrow$ & $\mathrm{NC}$ & $\mathrm{NC}$ & $\rightarrow$ & $\rightarrow$ & $\rightarrow$ & $\rightarrow$ \\
\hline $45^{\circ} \mathrm{Left}$ & $\rightarrow$ & $\mathrm{NC}$ & $\mathrm{NC}$ & $\rightarrow$ & $\mathrm{NC}$ & $\rightarrow$ & $\rightarrow$ \\
\hline $30^{\circ}$ Right & $\rightarrow$ & $\rightarrow$ & $\mathrm{NC}$ & $\rightarrow$ & $\mathrm{NC}$ & $\rightarrow$ & $\rightarrow$ \\
\hline $45^{\circ}$ Right & $\rightarrow$ & $\rightarrow$ & $\leftarrow$ & $\rightarrow$ & $\mathrm{NC}$ & $\rightarrow$ & $\rightarrow$ \\
\hline Back & $\rightarrow$ & $\mathrm{NC}$ & $\mathrm{NC}$ & $\rightarrow$ & $\mathrm{NC}$ & $\rightarrow$ & $\rightarrow$ \\
\hline Front & $\rightarrow$ & $\rightarrow$ & $\mathrm{NC}$ & $\rightarrow$ & $\mathrm{NC}$ & $\rightarrow$ & $\rightarrow$ \\
\hline
\end{tabular}


Table 3: Shift of Skewness value for different postures of all 6 subjects (two trials for subject 6). Skewness shifted towards more positive value: $(\rightarrow)$, No change: $(N C)$,

Skewness shifted towards more negative value: $(\rightarrow)$. Respective reference values are for upright position of head

\begin{tabular}{|c|c|c|c|c|c|c|c|}
\hline $\begin{array}{c}\text { Subject } \\
\text { Posture }\end{array}$ & $\mathbf{1}$ & $\mathbf{2}$ & $\mathbf{3}$ & $\mathbf{4}$ & $\mathbf{5}$ & $\mathbf{6 - a}$ & $\mathbf{6 - b}$ \\
\hline $\begin{array}{c}\text { Upright } \\
\text { (reference) }\end{array}$ & 0.56 & 3.13 & 2.55 & 1.04 & 0.56 & 4.11 & 1.57 \\
\hline $30^{\circ}$ Left & $\rightarrow$ & $\leftarrow$ & $\leftarrow$ & $\leftarrow$ & $\leftarrow$ & $\leftarrow$ & $\leftarrow$ \\
\hline $45^{\circ}$ Left & $\leftarrow$ & $\leftarrow$ & $\leftarrow$ & $\leftarrow$ & $\rightarrow$ & $\leftarrow$ & $\leftarrow$ \\
\hline $30^{\circ}$ Right & $\leftarrow$ & $\leftarrow$ & $\leftarrow$ & $\rightarrow$ & $\rightarrow$ & $\leftarrow$ & $\leftarrow$ \\
\hline $45^{\circ}$ Right & $\rightarrow$ & $\leftarrow$ & $\leftarrow$ & $\leftarrow$ & $\leftarrow$ & $\leftarrow$ & $\leftarrow$ \\
\hline Back & $\rightarrow$ & $\leftarrow$ & $\leftarrow$ & $\leftarrow$ & $\rightarrow$ & $\leftarrow$ & $\leftarrow$ \\
\hline Front & $\rightarrow$ & $\leftarrow$ & $\leftarrow$ & $\leftarrow$ & $\rightarrow$ & $\leftarrow$ & $\leftarrow$ \\
\hline
\end{tabular}

Table 4: Shift of lower half widths (lhw) at half maximum for different postures of all 6 subjects (two trials for subject 6). Shifted to higher value: $(\rightarrow)$, No change: $(N C)$, shifted to lower value: $(\rightarrow)$. Respective reference values are for upright position of head, in $\mathrm{ms}$.

\begin{tabular}{|c|c|c|c|c|c|c|c|}
\hline Subject & $\mathbf{1}$ & $\mathbf{2}$ & $\mathbf{3}$ & $\mathbf{4}$ & $\mathbf{5}$ & $\mathbf{6 - a}$ & $\mathbf{6 - b}$ \\
\hline $\begin{array}{c}\text { Upright } \\
\text { (reference) }\end{array}$ & 0.4 & 0.5 & 0.6 & 0.4 & 0.55 & 0.25 & 0.2 \\
\hline $30^{\circ}$ Left & $\rightarrow$ & $\leftarrow$ & $\rightarrow$ & $\rightarrow$ & $\rightarrow$ & $\rightarrow$ & $\rightarrow$ \\
\hline $45^{\circ}$ Left & $\rightarrow$ & $\leftarrow$ & $\rightarrow$ & $\mathrm{NC}$ & $\leftarrow$ & $\rightarrow$ & $\rightarrow$ \\
\hline $30^{\circ}$ Right & $\rightarrow$ & $\rightarrow$ & $\leftarrow$ & $\mathrm{NC}$ & $\leftarrow$ & $\rightarrow$ & $\rightarrow$ \\
\hline $45^{\circ}$ Right & $\rightarrow$ & $\leftarrow$ & $\leftarrow$ & $\rightarrow$ & $\leftarrow$ & $\rightarrow$ & $\rightarrow$ \\
\hline Back & $\leftarrow$ & $\leftarrow$ & $\rightarrow$ & $\rightarrow$ & $\leftarrow$ & $\rightarrow$ & $\rightarrow$ \\
\hline Front & $\leftarrow$ & $\leftarrow$ & $\leftarrow$ & $\rightarrow$ & $\rightarrow$ & $\rightarrow$ & $\rightarrow$ \\
\hline
\end{tabular}


Table 5: Shift of upper half widths at half maximum for different postures of all 6 subjects (two trials for subject 6). Shifted to higher value: $(\rightarrow)$, No change: $(N C)$, shifted to lower value: $(\rightarrow)$. Respective reference values are for upright position of head, in ms.

\begin{tabular}{|c|c|c|c|c|c|c|c|}
\hline Subject & $\mathbf{1}$ & $\mathbf{2}$ & $\mathbf{3}$ & $\mathbf{4}$ & $\mathbf{5}$ & $\mathbf{6 - a}$ & $\mathbf{6 - b}$ \\
\hline $\begin{array}{c}\text { Upright } \\
\text { (reference) }\end{array}$ & 0.65 & 0.35 & 0.35 & 1.35 & 0.44 & 0.3 & 0.4 \\
\hline $30^{\circ}$ Left & $\leftarrow$ & $\rightarrow$ & $\rightarrow$ & $\leftarrow$ & $\rightarrow$ & $\leftarrow$ & $\leftarrow$ \\
\hline $45^{\circ}$ Left & $\leftarrow$ & $\rightarrow$ & $\rightarrow$ & $\leftarrow$ & $\leftarrow$ & $\leftarrow$ & $\leftarrow$ \\
\hline $30^{\circ}$ Right & $\leftarrow$ & $\rightarrow$ & $\mathrm{NC}$ & $\leftarrow$ & $\rightarrow$ & $\leftarrow$ & $\leftarrow$ \\
\hline $45^{\circ}$ Right & $\rightarrow$ & $\rightarrow$ & $\rightarrow$ & $\leftarrow$ & $\rightarrow$ & $\mathrm{NC}$ & $\leftarrow$ \\
\hline Back & $\leftarrow$ & $\rightarrow$ & $\leftarrow$ & $\leftarrow$ & $\leftarrow$ & $\leftarrow$ & $\leftarrow$ \\
\hline Front & $\leftarrow$ & $\mathrm{NC}$ & $\mathrm{NC}$ & $\leftarrow$ & $\leftarrow$ & $\mathrm{NC}$ & $\leftarrow$ \\
\hline
\end{tabular}

Table 3 shows direction of shifts of the Skewness in all the 6 subjects. For Subject 6 there are two repeat measurements, as before (6-a, 6-b) and they appear to be consistent. Table 4 shows the lower half widths at half maximum as defined in Figure 1 for all the 6 subjects, with repeat trials for subject 6 as before (6a, 6-b) while Table 5 shows the upper half widths at half maximum. As can be seen, the shift in the value of the parameter is mixed, but for the same subject, the directions are mostly the same. Again, the shifts in the two trials on subject 6 appear to be consistent.

\section{DISCUSSIONS}

During previous experiments, researchers of our extended group found that repeated DFLs obtained from a particular nerve in the upper limb of a subject correlate well to each other if these are taken in a single session with the subject remaining in the same seating position. However, on a different session on a different day the correlations were not good sometimes. This gave rise to the idea that possibly posture of the subject, particularly of the head and neck, was affecting the DFL. Therefore it was necessary to understand this phenomenon well and the previous work (Rahman 2007) was carried out to observe the effect of postures of head and neck on DFL. Comparing the DFLs obtained with the subject sitting normally, i.e. in a straight sitting position with the DFLs obtained by tilting head towards left, right, back and front directions, it was found that, patterns of DFL changes for different postures of head and neck, but the changes of patterns of DFL were not systematic to draw any conclusion. The present work was taken up to verify the earlier result, but by improving the methodology. In the previous work the angle of bending of head in different directions were not specified, it could have varied from trial to trial. So in this present work, the changes of patterns of DFLs were studied for a few specific postures of head, at 
certain fixed angles. To obtain the DFLs, evoked M-responses were recorded from the Thenar muscle by stimulating the median nerve at the wrist.

It should be noted that the F-latency depends on the length of the arm of a person and the conduction velocity of the peripheral nerve (here, median nerve). However, the pattern of DFL is expected to remain the same.

Table 2 shows that compared to DFL's for upright position of head the Peak of DFL shifted towards higher latency, or there was no change in some cases, for all other postures. This happened for all the subjects.

Table 3 shows that the Skewness value mostly shifted towards more negative values for different postures of all the six subjects. This agrees with the peak shift of DFL as observed in Table 2 as the tail of the curve extends towards the lower values when the peak moves towards higher values contributing to a negative shift in skewness. Tables 4 and 5 shows that the lower half width (lhw) values mostly increased while the upper half width (uhw) mostly decreased with changed posture. Again this agrees with the change of skewness observed.

However, it needs to be noted that based on DFL in the upright position [Rabbani et al 2014, Chowdhury et al 2014, Rahman et al 2014] most of the subjects had cervical radiculopathy or myelopathy (compression of spinal nerve or of the spinal cord) and therefore, changes occurring in DFL due to changes of head posture would be complex. However, the repeatability of the measurements on subject 6 points to the strength of DFL.

Therefore, from the above discussion we come to the conclusion, that postures of head has significant effect on DFL.

In this present work, temperature effect on DFL was neglected, because the experiment was carried out within a short span of time, during which the atmospheric temperature did not change significantly, but the effect of this parameter can be studied in future work. This work also had a small number of participants. The same study should be performed on a large number of subjects when the results will be established on a stronger footing.

The effect of spinal traction may also be studied in the case of cervical spondylosis patients, to see that whether there occur any systematic change in DFL before and after the traction. All these studies may open up a whole new avenue in neural investigation and diagnosis and increase the area of application of this new DFL technique.

\section{Acknowledgment}

The authors would like to thank the International Science Programme (ISP) of Uppsala University, Sweden for part financial support. 


\section{REFERENCES}

Alam MJ and Rabbani KS, 2010. Possible detection of cervical spondylotic neuropathy using Distribution of Flatency (DFL), a new neurophysiological parameter, BMC Research Notes, 3:112, <http://www.biomedcentral.com/1756-0500/3/112>.

Chowdhury EA, Hussain MZ, Rahman MO and Rabbani KS, 2014. A double blind study to evaluate the efficacy of distribution of F- latency (DFL) in the detection of cervical radiculopathy and myelopathy, Bangladesh Journal of Medical Physics, 7: 46-55.

Hodgkin AL, 1939. The relation between conduction velocity and the electrical resistance outside a nerve fibre, $J$. Physiol. 94: 560-570.

Hossain MI, Chowdhury EA, Mamun AA, Salam A, Baig TN and Rabbani KS, 2011. Use of Distribution of FLatency (DFL) in the detection of cervical spondylotic neuropathy, Bangladesh Journal of Medical Physics, 4: $37-42$.

Rabbani KS, Alam MJ and Salam MA, 2007. Frequency Distribution of F-Latencies (DFL) has physiological significance and gives Distribution of Conduction Velocity (DCV) of motor nerve fibres with implications for diagnosis. J of Biol Phys. (Springer), 33: 291-303. doi:10.1007/s10867-008-9071-6.

Rabbani KS, 2011. Hypotheses to Explain the Occurrence of Multiple Peaks of DFL in Nerve Conduction Measurement. Bangladesh Journal of Medical Physics, 4: 27-36.

Rabbani KS, Yassin N and Lo YL, 2014. Identification of Cervical Spondylotic Radiculo-Myelopathy using Distribution of F-Latency (DFL), a new nerve conduction parameter. Bangladesh Journal of Medical Physics, 7: 34-45.

Rahman MM, 2007. Study of the Distribution of F-Latency (DFL) for different postures of our head and neck, M.Sc. thesis, Dept. of Physics, University of Dhaka.

Rahman MO and Rabbani KS, 2014. Distribution of conduction velocity (DCV) from measured F-wave latency for detection of cervical spondylotic radiculopathy and myelopathy (CRM), Bangladesh Journal of Medical Physics, 7: 56-66. 